\title{
Do People Like Working With Computers More than Human Beings?
}

\author{
Marek N. Posard \\ University of Maryland \\ R. Gordon Rinderknecht \\ University of Maryland
}

Corresponding Author:

Marek N. Posard

University of Maryland

(202)-527-9344

mposard@gmail.com 


\begin{abstract}
This paper incorporates the concept of mindlessness from research on human-computer interactions with social exchange theory from sociology. We find that participants behaved no differently toward human or computerized partners during a repeated standard trust game. Despite exhibiting similar behaviors with these partners, participants believed that computers were more likely to share their interests during this game than humans. These participants also reported higher levels of commitment with computerized partners than human partners. Our results suggest that asking about social constructs (i.e. commitment) will break mindlessness in human-computer interactions. These results also highlight a disconnect between individual behaviors and their perceptions during human-computer interactions. We conclude that telling participants their partners are computers may actually improve their perceptions of interactions after they occur.
\end{abstract}

Do People Like Working With Computers More than Human Beings? 
Samantha West has a pleasant-sounding voice on the telephone (M iller \& Nicks, 2013). She is confident and coherent when talking with new clients. Her persona is bubbly and she exhibits noticeable excitement when selling her company's product to people as a telemarketer. Samantha is a model salesperson, except that she is not a person at all. She is a computer that talks to potential clients about buying insurance products on the phone as if she was a real human being. She will even lie to customers and deny that she's a robot (M iller \& Nicks, 2013). This example highlights a change in the way that humans and computers interact with each other in various facets of life. The present study examines how people perceive their interactions with partners that are computers or human beings.

The pace of innovation in technology appears to be changing the way that humans communicate with their computers. In the 1960s and 1970s, humans had to learn complicated code to instruct a mainframe computer to produce specific types of output (e.g. analysis of variance outputs). In the 1980s and 1990s, graphics would simplify these complex codes for the average consumer who used a personal computer (e.g. Windows 3.1). Today, computers have the capacity to interact with humans before these users clearly know what they may want their machine to do for them.

For example, the IBM computer, named Watson, beat two human contents on the game show Jeopardy!, while an estimated 21-million people watched it respond to questions from another person. The Apple iPhone and iPad mobile devices have voice-recognition software, named Siri, that responds to a variety of verbal questions or statements from human users. The romantic movie, Her, won an Academy Award for Best Picture in 2013 by telling the story of a 
man falling in love with his computer operating system. Simply put, it appears that computers have become more social with the advancement of information technology.

This paper applies social exchange theory from sociology to the study of humancomputer interactions. Social exchange theory explains how the pattern of interactions between people affects their perceptions of each other in groups. The social exchangetheoretic approach traditionally defines a group as two or more humans that work together on some collective task. We broaden this definition of a "group" to include humans and computers with artificial intelligence capabilities. We then use an experiment to test if the behaviors and attitudes of participants will significantly vary when their partners are human beings or computers. Our results show that asking people about their perceptions of interactions can "break" their mindlessness during human-computer interactions, even though they exhibit similar behavior towards human and computerized partners during an exchange task. This break, in turn, can inflate user perceptions of their interactions with computers in contrast to humans.

\section{Theoretical Background}

\subsection{Social Exchange Theory}

Social exchange is the reciprocal transfer of resources between two or more people during a repeated number of interactions (Emerson, 1976). Theories of social exchange explain how these transfers affect the attitudes and behaviors of people in groups. The social exchange-theoretic framework views exchange outcomes as interdependent (Emerson, 1976; 
Homans, 1958). Thus, the outcomes of past exchanges can affect how individuals behave in future exchange relationships.

There is considerable evidence from experiments in sociology that enduring patterns of social exchange affect the feelings and beliefs of those housed within groups (Lawler \& Yoon, 1996; 1998; M olm, 1994; M olm, Takahashi, \& Peterson, 2000). For example, studies find that people experience positive feelings when they gain resources for themselves during social exchange (Lawler \& Yoon, 1996; 1998). When they gain resources during several periods of social exchange, these experiments find that positive feelings will affect the formation of cohesion in groups (Lawler \& Yoon, 1996; 1998; Lawler, Thye, \& Yoon, 2008).

Other research has found that enduring patterns of exchange can influence the degree of cohesion that exists in small groups (M olm, 1994; M olm, Takahashi, \& Peterson, 2000). This research proposed that risk of non-reciprocity is a necessary condition for trust to form between people in groups (M olm, Takahashi, \& Peterson, 2000). When group members begin to recognize a pattern of reciprocity during social exchange, experiments find that perceptions of cohesion emerge in these groups (M olm, Takahashi, \& Peterson, 2000). Thus, a stable pattern of reciprocity that social exchange produces over time can lead individuals to trust their partners that, in turn, produce group cohesion.

The definition of "group" by social exchange theorists is typically defined as two or more humans that work together on some collective task. We propose broadening this treatment to include groups where humans work with partners that are computers. With the rapid advancement of technology, computers now have the capacity to socially interact with people without direct inputs from users. These social capacities raise questions about the degree that 
the interpersonal dynamics known to affect the attitudes and behaviors of humans also exists when humans work with partners that are computers.

\subsection{Social Computing and M indlessness}

Mindfulness refers to the over-reliance on habits from past experiences that one applies to their new experiences (Langer, 1992). Such over-reliance leads one to rely on preexisting scripts that may not take into account some important qualities of the individual and the situation. Numerous studies suggest that people mindlessly rely on "scripts" - typically used for interpersonal interactions - when they interact with computers (Nass \& Moon, 2000).

For example, Nass and M oon (2000) use the concept of mindlessness to interpret research finding that people tended to evaluate a computer with a man's voice as more competent than a computer with a woman's voice, unless the computer was talking about stereotypically feminine subjects (Nass, M oon, \& Green, 1997). There was no difference in the dialogues used by these two machines, yet participants appear to have continued to rely on scripts imported from interactions with humans without considering the scripts' irrelevance to computers. Similarly, Posard (2014) found that evaluations of a computer's performance on a group task did not significantly change when the machine was named "James" or "Julie," however, these participants did estimate the former costs significantly more to purchase than the female machine. This finding suggests that participants rely on the social "script" of gender when asked to formulate cost estimates of their computers.

Nass and M oon (2000) also argued that the mindless applications of scripts and stereotypes can alter perceptions of machines when the differences are based on in-group 
ethnic identity (Nass, Isbister, \& Lee, in press) and group identity based on minimal, arbitrary differences (Nass, Fogg, \& Moon, 1996). This application of mindlessness also extended to normative behavior, where Nass and M oon (2000) argued that the norm of reciprocity (Gouldner, 1960) extends to people's interactions with "helpful" computers (Fogg \& Nass, 1997). Nass and M oon (2000) observe that throughout their research they have never encountered a participant who verbally declared that computers should be understood or treated like humans-yet, across many studies, people have been shown to often behave as if human scripts and stereotypes applied equally well to computers.

In the present study, we ask two important questions for research on human-computer interaction. First, what are the conditions that break mindlessness for people during these interactions? Second, could breaking this mindlessness have benefits for the way that people perceive their interactions with computers?

\section{Predictions}

Drawing from research on mindlessness that suggests people treat computers as if they are humans across a wide range of situations (Nass \& M oon, 2000), we expect participants to exhibit similar behaviors toward partners that are described as being humans or computers, unless sufficiently motivated to examine the identities of their partners. Shank (2012) suggested that disrupting this process of mindlessness by having participants encounter normatively unexpected behaviors from their partners would provide such motivation. This argument leads to our first hypothesis, predicting that participants will behave no differently toward human or computerized partners who behave generously toward them because 
generosity is normatively expected in this situation. We define generous partners as those who give the vast majority of their resources to participants during each period of exchange (i.e. they gave $95 \%$ or more of their resources to participants). We make the following prediction:

Hypothesis 1: Participants will give equal amount of resources to human or computerized partners who behave generously toward them.

Shank (2012) expected that a partner's coercive behaviors (that he defined as the use of punishments to gain compliance) would disrupt participants' mindlessness, which would lead participants to examine the identity of their partner. When the partner is a computer, participants might conclude that a machine cannot intentionally behave in an unjust manner and is therefore less deserving of retribution. Although Shank (2012) proposed several mechanisms to explain his findings, his results largely support what was predicted by his treatment of mindlessness, and we expect a similar process to occur when participants interact with a selfish partner. Hypothesis 2 predicts that selfish behaviors by partners will "break" the mindlessness of participants because such behavior is not normatively expected, and that a closer evaluation of the partner's identity may lead participants to behave more generously toward partners that are described as computers instead of humans. This is because it may be harder to apply human characteristics, such as selfishness, to computers. We define selfish partners as those who keep the vast majority of their resources for themselves during each 
period of exchange (i.e. they keep 95\% or more of their resources for themselves instead of giving it to participants). We make the following prediction:

Hypothesis 2: Participants will give more resources to a computerized rather than human partner who behaves selfishly toward them.

It is conceivable that selfish behaviors may not be sufficiently unexpected to "break" participants' mindlessness, or that an examination of the partner's identity will still result in a similar degree of trusting behavior. However, we expect that directly asking participants to assess their partner's motivations will break their state of mindlessly responding to social cues. Assessing a partner's motives should require an examination of the partner's identity, and the computer identity may make it difficult for one to perceive selfish intentions even when a computer is acting selfishly, which should boost perceptions of shared interest and, potentially, one's commitment to a computer partner relative to a human partner. This is because computers may be perceived as mere devices that users have difficulty ascribing human characteristics to, perhaps because we do not take the intentions of programmers into consideration (Reeves \& Nass, 1996). Based on this reasoning, we predict that participants will report more positive evaluations of their relationships with partners that are computers instead of humans: 
Hypothesis 3: Participants will believe their interests were more aligned with a partner that is a computer rather than a human being.

Hypothesis 4: Participants will report higher levels of commitment to their partner when this partner is a computer rather than a human being.

\section{Method}

\subsection{Sample and Procedure}

This study recruited 124 adult participants ( 80 women and 44 men) from Amazon's Mechanical Turk Internet marketplace to complete a group task with another human-being or a computerized partner who behaved selfishly or generously during the group task. The study guaranteed participants $\$ 1.50$ for about 15 -minutes of their time to complete this task. One manipulation check asked participants if they saw a picture of a computer or human on their screens during the group task. A second manipulation check asked them to rate how generous their computerized or human partner behaved. We excluded ten participants who failed these two manipulation checks, leaving a final sample size of 114. The results from a Pearson's chi ${ }^{2}$ test show no significant differences in the sample sizes between each of the experimental conditions $\left(\mathrm{Chi}^{2}=146 ; \mathrm{p}<703\right){ }^{1}$

\footnotetext{
${ }^{1}$ Our final sample had 26 participants with a selfish human partner, 30 participants with a selfish computerized partner, 29 participants with a generous human partner, and 29 participants with a generous partner that was a computer.
} 
Participants gave their consent to participate in this study, completed a demographic questionnaire, followed by five rounds of an exchange task, and concluded the study by completing a short questionnaire about their perceptions of this task. The study instructions told participants the tokens they earned in this study would equal the number of raffle tickets they would receive. The participant who wins this raffle would receive $\$ 5.00$ in addition to the $\$ 1.50$ they received for their participation. ${ }^{2}$

The exchange task in this study was a version of the standard trust game (Berg, Dickhaut, \& M cCabe, 1995). For each round of exchange, the study would give 20 tokens to the participant and another 20 tokens to their partner. Both partners would simultaneously decide to give none, some, or all of their tokens to the other person. This task would automatically double whatever amount one partner gave to the other. The earnings for this period would be the amount that participants kept in addition to the doubled amount they received from their partners.

To illustrate, imagine a group of two people named Jack and Jill. The exchange task would give 20 tokens to Jack and another 20 tokens to Jill. Let us say that Jack decides to keep 15 tokens for himself and gives Jill five of his tokens. The task would double this amount so that Jill would receive 10 tokens. Jill decides to give Jack 10 tokens and keeps the remaining 10 tokens for herself. This task would double this amount so Jack actually receives 20 tokens from his partner. The period would conclude with Jill earning 20 tokens (the 10 she kept plus the 10

\footnotetext{
${ }^{2}$ The instructions told participants with human partners that they would compete in separate raffles from their partner. This reduced the salience of competition between participants and their human partners that may not occur with computerized partners who could not win the raffle. The results from a one way analysis of variance did not find significant differences in generosity during the exchange task for participants that believed their partner was a human or computer (chi ${ }^{2}=.48 ; p<49$ ).
} 
that she received from Jack). For Jack, his earnings would equal 35 tokens (the 15 he kept plus the 20 that Jill transferred to him). Then, the new period would begin with Jack and Jill each receiving another 20 tokens.

The experiment told participants they would complete seven periods of exchange with the same partner. In reality, participants only completed five periods. This prevented bias from "end effects." If participants know the task is about to conclude, some participants may choose to behave less generously towards the end of an exchange task independent of our manipulations. Consequently, end effects may alter participant responses on subsequent questions that ask about shared interests and commitment in their groups.

\subsection{M anipulations}

Participants completed the entire study using Qualtrics web-based software. This software randomly assigned participants to one of the following four conditions: (i) Human partners that would behave generously in the exchange task, (ii) Computerized partners that would behave generously during this task, (iii) Human partners that behaved selfishly during the exchange task, or (iv) Computerized partners that behaved selfishly during this task. Unbeknown to participants, the partners were computer algorithms that would give the same amounts if they were computers or humans.

Our study had two manipulation checks. First, we asked participants if they saw an image of a human or computer mainframe on their own computer screens. The question asked, "Pick the image that you saw on your computer screen during this experiment." Participants could choose one of two images: a human silhouette or a computer server. One of these two 
images was displayed on the screen of participants in our study. We excluded eight participants who incorrectly selected the image of a computer when they were in conditions that displayed images of a computer server or those who selected the computer server image despite being in conditions that displayed a human silhouette image.

The first manipulation check only measures whether or not participants noticed a defining characteristic of their partner: if this partner was a computer or human-being. It does not, however, measure the degree to which participants believed they were actually working with a computerized or human partner. ${ }^{3}$ To address this concern, we also asked participants one of two questions toward the end of the experiment: "Did you believe your partner was a real person?" or "Did you believe your computerized partner was a real computer?" Participants could select "Yes" or "No" as their answers. We constructed a dichotomous variable where 1 represents those who believed our manipulation was real and 0 are those who answered no to these questions. The results from a Pearson's Chi ${ }^{2}$ test showed no significant differences between conditions for participants that selected "Yes" or "No" as answers to this question $\left(\mathrm{Chi}^{2}=2.41 ; \mathrm{p}<0.49\right.$, two-tailed). We found similar non-significant differences when comparing answer choices of participants with computerized or human partners $\left(\mathrm{Chi}^{2}=1.24\right.$; $p \varangle 0.27$, two-tailed), and those with generous or selfish partners ( $\mathrm{Chi}^{2}=0.29 ; \mathrm{p} \varangle 0.59$, twotailed).

Second, we asked participants to rate how generous their computerized or human partner behaved toward them. Participants used a six-point scale that ranged from "Strongly Disagree" to "Strongly Agree." Two participants choose "Agree" or "Strongly Agree" to this

\footnotetext{
${ }^{3}$ We thank an anonymous reviewer for making this point to us.
} 
question, despite having partners that we pre-programmed to behave selfishly. We removed these two participants from our final sample. ${ }^{4}$ We discuss the details for each of these manipulations below.

\subsubsection{Computerized or Human Partner}

After participants read the study instructions, the study displayed the image of a "spinning wheel" above the text, "One moment please. We are connecting to your partner." The study displayed this progress screen for 10 seconds and automatically advanced participants to the next screen. For half of the participants in our study, this screen told participants their partner is a real person who, like them, is a registered user of Mechanical Turk. It said their partner is reading the exact same instructions as them and, like them, will have the opportunity to earn tokens for a chance to win a raffle prize.

The instructions also told participants the study would hold separate raffles for them and their partners. So, the tokens that participants earned would not affect the chances of their partner winning a raffle prize. The instructions told participants they would never meet nor communicate directly with their partner either. Further, the study stated that partners would not know any personal information about each other (e.g. their M echanical Turk username, their MTurk user rating, demographic background characteristics, or e-mail address).

The Qualtrics software randomly assigned other participants to complete the same exchange task with a computerized partner. After participants saw the "spinning wheel" image for ten seconds, this software displayed a new screen to the other half of participants that

\footnotetext{
${ }^{4}$ Removing these two participants did not significantly affect our results.
} 
stated: “Unfortunately, we could not find a human partner for this task. Would you like to continue with a computerized partner? If you agree, please click the selection below and click next." Participants had to select a box next to the text, "I will continue with a computerized partner." This led participants to the instructions screen for computerized partners.

These instructions told participants their partner is a computer that can read, understand, and analyze unstructured data. The study continued by telling participants this computer represents a first step into cognitive systems, a new era of computer. It also stated this computer uses programmatic computing plus the combination of three additional capabilities: natural language processes, hypothesis generation and evaluations, and dynamic learning. The instructions explained that, through repeated use, this computer literally gets smarter by tracking feedback from its users and learning from both successes and failures.

\section{[Insert Figure 1 Here]}

Figure 1 displays the image that participants saw during the group task with a computerized or human partner. Participants with a human partner saw a silhouette image of a person. The exchange task referred to this person as the partner of participants. In comparison, participants with a computerized partner saw an image of a computer server. The exchange task referred to this partner as the computerized partner of participants.

\subsubsection{Generous or Selfish Partners}


Participants had computerized or human partners that were, unbeknown to them, computer algorithms that would behave generously or selfishly during the group task. These pre-programmed behaviors were the same for computerized or human partners. For partners that behaved generously, the algorithm would automatically give partners 20 tokens for the first three rounds of exchange. This algorithm gave partners 19 tokens in the fourth round, and then gave 20 tokens in the fifth round of exchange. The Qualtrics software would delay these decisions for several seconds to increase the believability of participants in this study. The timing of this delay was the same for participants that had a computerized or human partner.

For those assigned selfish partners, the Qualtrics software had the computerized or human partner give participants zero tokens in the first three rounds of exchange. This selfish partner gave one token to participants in the fourth round and then gave them zero tokens in the fifth round of exchange. This study used the same timing delays across each of the experimental conditions.

\subsection{Dependent Measures}

Generosity. - This study has three dependent measures of interest. The first measure is generosity by participants toward a computerized or human partner. This measure is an index of the amounts that participants gave to their partners in the five rounds of exchange. The Cronbach alpha measure of inter-reliability for this index is $0.91(M=10.59, S D=6.57)$.

Shared Interests. - The second dependent measure is the degree to which participants believed they shared the same interests as their partners. This measure is an index of two questions with a Cronbach's alpha of $0.84(\mathrm{M}=3.35 ; \mathrm{SD}=1.83)$. One question asked 
participants, "On the whole, do you think your interests were in conflict with your [computerized] partner, or were your interests in agreement?" Participants answered using a six-point scale that ranged from "Very conflicting" to "Very much in agreement." The second question from this index asked participants, "Would you say that the motives of your [computerized] partner were generally cooperative or competitive?" Participants used a sixpoint scale that ranged from "Very competitive" to "Very cooperative."

Commitment to Partner. - The last dependent measure is participant commitment to their partner. This measure is an index of three questions with a Cronbach's alpha of $0.81(\mathrm{M}=$ 3.60; SD =1.49). The first question asked participants, "How much do you agree with the statement: I feel that my [computerized] partner and I worked well together." Participants used a six-point scale that ranged from "Strongly disagree" to "Strongly agree." The second question asked participants, "How committed were you to your [computerized] partner? The answer choices ranged along a six-point scale from "Very uncommitted" to "Very committed." The last question asked, "How would you describe your general feelings toward your [computerized] partner?" The answer choices ranged from "Very negative" to "Very positive" using a six-point scale. 


\section{Results}

\subsection{Generosity Toward Partners Behaving Generously}

Hypothesis 1 predicts that participants would give an equal amount of tokens to computerized partners and human beings. The results from an analysis of covariance (ANCOVA) model in Table 1 support this prediction. Participants did not give significantly more tokens to computerized partners than human partners ( $F=.13 ; p<72$; two-tailed).

\section{[Insert Table 1 Here]}

\subsection{Generosity Toward Partners Behaving Selfishly}

Hypothesis 2 predicts that participants would give more resources to selfish partners that are computers than selfish human partners. We did not find support for this prediction. Table 1 also shows no significant interaction effect between a computerized or human partner and whether this partner behaved generously or selfishly $(F=0.44 ; p<51$, two-tailed). This ANCOVA model was significant overall ( $F=18.09 ; p<001$, two-tailed). Thus, we find that selfish or generous behavior may not "break" the mindlessness of participants during humancomputer interactions.

\subsection{Perceptions of Shared Interests}

Hypothesis 3 predicts that participants will be more likely to believe they share interests with computerized partners than human partners. The results in Table 2 support this 
prediction. Participants tended to report more shared interests with computer partners than they shared with human partners ( $F=3.98 ; p<05$, two-tailed). This model also shows that shared interests were higher for partners that were more generous than selfish ( $F=464.77$; $p<001$, two-tailed). There was no significant interaction effect between partner type and partner's behaviors ( $F=0.8 ; p \varangle 0.78$, two-tailed). The overall ANCOVA model in Table 2 was also statistically significant $(F=155.81 ; p<001$, two-tailed).

\section{[Insert Table 2 Here]}

\subsection{Perceptions of Commitment to Partner}

Hypothesis 5 predicts that participants will report a higher degree of commitment towards a computerized partner than one that is a human. The results in Table 3 support this prediction. Participants reported higher levels of commitment when their partner was a computer rather than a human ( $F=4.41 ; p<04$, two-tailed $)$.

\section{[Insert Table 3 Here]}

Similar to our previous findings, Table 3 shows that generosity significantly affects commitment between participants and their partners ( $F=181.34 ; \mathrm{p}<001$, two-tailed). The table also shows no statistically significant interaction effect between type of partner and the 
generosity this partner exhibited toward participants ( $F=0.03 ; p<87$, two-tailed). The overall ANCOVA model in Table 3 was also statistically significant $(F=61.47 ; p<001)$.

\section{Review of Findings}

We present evidence that asking people about social constructs (i.e. shared interests and commitment) may have the capacity to "break" their mindlessness during humancomputer interactions. Overall, participant behaviors did not significantly differ when they completed a standard trust game with partners they believed were computers or real human beings. However, the way these participants evaluated their interactions with computers did significantly vary by the type of partner we assigned to them. Participants reported that the interests of a computer were more aligned with their own than an actual human partner. These same participants also reported higher levels of commitment toward their computerized partners than human partners.

\section{[Insert Figure 2 Here]}

Figure 2 displays the marginal effects of partner type (computer versus human partner) from the ANCOVA models that predict participants' perceived sense of shared interests and commitment with their partners. Despite the fact that participants behaved in a similar manner 
toward partners that were humans or computers, Figure 2 shows significant differences in the way that participants perceived their interactions with these partners. Merely telling participants their partner was a computer instead of a human, and showing them an image of a computer instead of a human silhouette, significantly altered how they perceived their interactions with computers versus humans. These results indicate that participants organized their retrospective views of their interactions with computers in different ways in comparison to their views of human partners.

\section{Discussion and Conclusion}

Social exchange theory explains how enduring patterns of interactions affect the attitudes and behaviors of people. Much of the research on social exchange theory defines a group as two or more people. We broaden this definition by examining how these interaction patterns affect dynamics in groups where humans work with computers. Using the social exchange theoretic-approach, we then examine when people decide to mindlessly treat their interactions with computers as if these partners were humans.

Our findings have several implications for advancing theory and applied research on human-computer interactions, but these findings are not without limitations. Our results incorporate the concept of mindlessness from human-computer interaction research into social exchange theory from sociology. We found support for hypothesis 1 , however we did not find support for hypotheses 2 . Overall, these findings show participants behaving no differently toward partners that were humans versus computers. This result may be a function of the sample we used in our study-workers from Amazon's M echanical Turk. This sample might 
have been overexposed to exchange research in ways that make it difficult to find effects in exchange tasks (Rand et al., 2014). We are currently unable to say if such overexposure is responsible for the success of hypothesis 1 and the failure of hypothesis 2 .

We found support for hypothesis 3 and 4, both of which relate to participants' perceptions of their partner. Hypothesis 3 predicted that participants would perceive their interests to be more aligned with computer partners, and hypothesis 4 expected participants to perceive themselves to be more committed to computer partners. Both of these hypotheses were based on the difficulty people have applying human characteristics to computers-in this case, selfishness. Although the failure of hypothesis 2 is difficult to interpret, its failure, along with support for hypotheses 3 and 4, are consistent with the findings of Parise et al. (1999). A post-experimental survey used by Parise at al. (1999) found that participants felt a greater sense of partnership (mutual liking and trustw orthiness) with computer partners, regardless of appearance, than human partners following an exchange requiring trust. This sense of partnership after the exchange, however, did not correspond to an increase in actual trusting behavior during the exchange. This was not expected by Parise et. al. (1999) and was only given limited attention within their discussion. Our consistent findings, using a different design, research population, and over a decade of cultural change regarding our interactions with computers, suggest that an enduring process may be affecting our interactions with computers that, in certain circumstances, will lead us to feel a greater sense of partnership with computers than people.

Returning to the example of the computer named Samantha West from our introduction section, the results in this paper suggest that people will not treat Samantha West 
any differently because she is a computer. In fact, they may perceive these interactions more positively because she is a computer instead of a human being. Our findings have applications for the design of artificial intelligence (Al) systems: telling end-users their partners are computers instead of hiding this fact could improve how these users perceive their interactions with Al technology, especially if the interaction contains a threat of exploitation or distrustful behavior. Simply put, the fact that Samantha West is a computer - and not a human - may add to the charm of this machine as perceived by its customers. 


\section{REFERENCES}

Berg, J., Dickhaut, J., \& M cCabe, K. (1995). Trust, reciprocity, and social history. Games and economic behavior, 10, 122-142.

Emerson, R. M. (1976). Social exchange theory. Annual review of sociology, 2, 335-362.

Fogg, B. J., \& Nass, C. (1997). Do users reciprocate to computers? In Proceedings of the CHI Conference. (pp. 331-332). New York, NY: Association of Computing Machinery.

Gouldner, A. W. (1960). The norm of reciprocity: A preliminary statement. American Sociological Review, 25, 161-178.

Homans, G. C. (1958). Social behavior as exchange. American journal of sociology, 63, 597-606.

Langer, E. J. (1992). M atters of mind: M indfullness/mindlessness in perspective. Consciousness and Cognition, 1, 289-305.

Lawler, E. J., \& Yoon, J. (1998). Network structure and emotion in exchange relations. American Sociological Review, 871-894.

Lawler, E. J., \& Yoon, J. (1996). Commitment in exchange relations: Test of a theory of relational cohesion. American Sociological Review, 89-108.

Lawler, E. J., Thye, S. R., \& Yoon, J. (2008). Social exchange and micro social order. American Sociological Review, 73, 519-542.

Miller, Z, D. Nicks. (2013). M eet the robot telemarketer who denies she is a robot. Time Magazine. Retrieved 16 April 2015, from http:// newsfeed.time.com/2013/12/10/meet-therobot-telemarketer-who-denies-shes-a-robot/.

M olm, L. D., Takahashi, N., \& Peterson, G. (2000). Risk and trust in social exchange: An experimental test of a classical proposition. American Journal of Sociology, 105, 1396-1427.

M olm, L. D. (1994). Dependence and risk: Transforming the structure of social exchange. Social Psychology Quarterly, 57, 163-176.

Nass, C., Fogg, B. J., \& M oon, Y. (1996). Can computers be teammates? International Journal of Human-Computer Studies, 45, 669-678.

Nass, C., \& Moon, Y. (2000). Machines and M indlessness: Social Responses to Computers, Journal of Social Issues, 56, 81-103. 
Nass, C., M oon, Y., \& Green, N. (1997). Are computers gender-neutral? Gender stereotypic responses to computers. Journal of Applied Social Psychology, 27, 864-876.

Nass, C., Isbister, K., \& Lee, E.-J. (in press). Truth is beauty: Researching conversational agents. In J. Castells (Ed.), Embodied conversational agents. Cambridge, M A: MIT Press.

Parise, S., Kiesler, S., Sproull, L., \& Waters, K. (1999). Cooperating with life-like interface agents. Computers in Human Behavior, 15, 123-142.

Posard, M. N. (2014). Status processes in human-computer interactions: Does gender matter? Computers in Human Behavior, 37, 189-195.

Reeves, B., \& Nass, C. (1996). The M edia Equation: How People Treat Computers, Television, and New Media Like Real People and Places. New York, NY: CSLI.

Shank, D. B. (2012). Perceived Justice and Reactions to Coercive Computers. Sociological Forum, 27, 372-391.

Rand, D. G., Peysakhovich, A., Kraft-Todd, G. T., Newman, G. E., Wurzbacher, O., Nowak, M. A., $\&$ Greene, J. D. (2014.) Social heuristics shape intuitive cooperation. Nature Communications, 5, $1-12$. 


\section{Figures}

Figure 1. Images used for $M$ anipulations

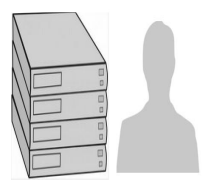

Computerized Partner Human Partner 
4

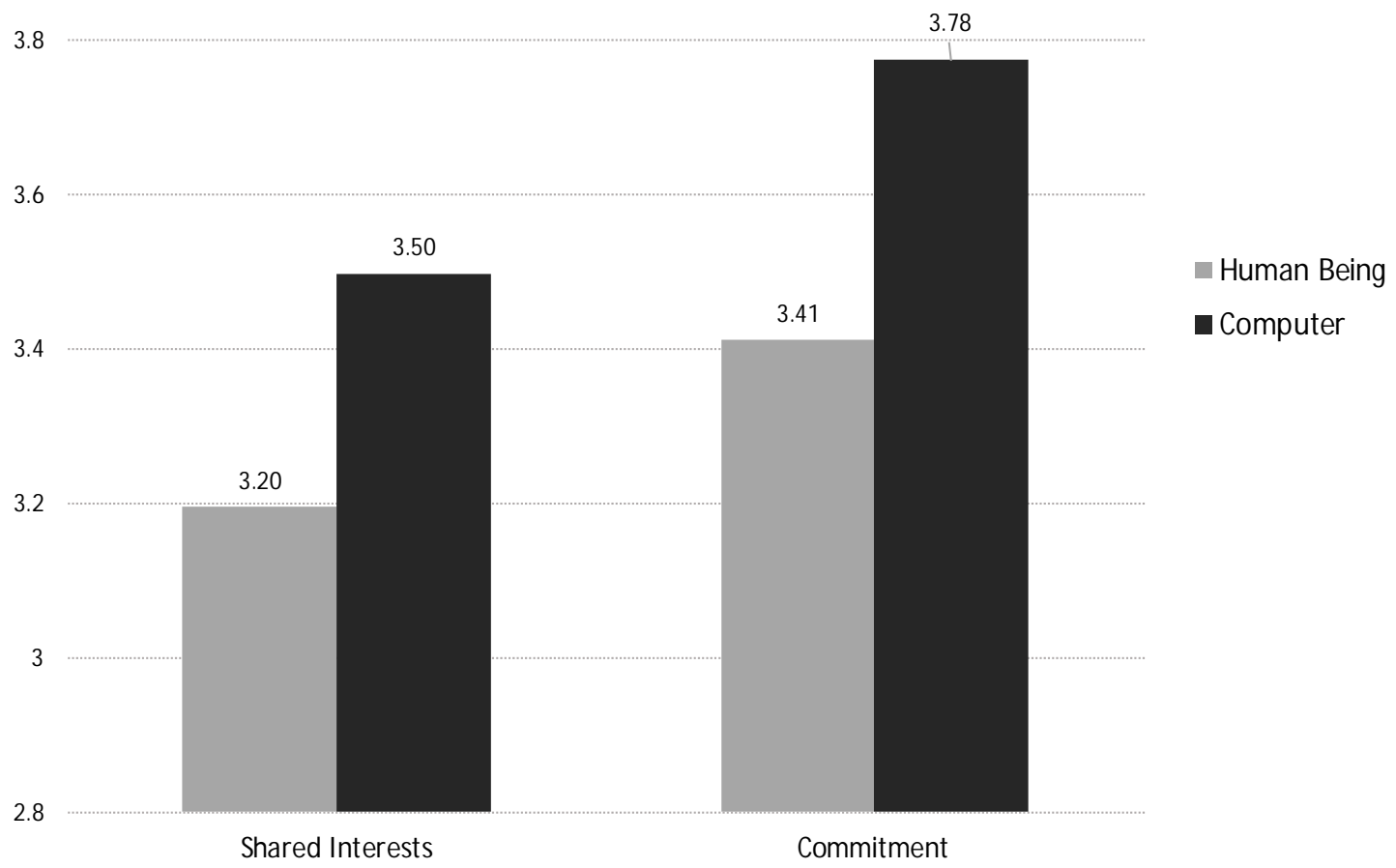

Figure 2. Marginal Effects for M easures of Shared Interests and Commitment by Partner Type 


\section{Tables}

Table 1. ANCOVA model Predicting Generosity by Participants

\begin{tabular}{|l|l|l|l|l|}
\hline \multicolumn{1}{|c|}{ Source } & \multicolumn{1}{|c|}{ SS } & \multicolumn{1}{|c|}{ df } & \multicolumn{1}{c|}{ MS } & \multicolumn{1}{|c|}{ F } \\
\hline Model & 1607.35 & 3 & 535.78 & $18.06 * * *$ \\
\hline Type of Partner: Computer (versus Human) & 3.77 & 1 & 3.77 & 0.13 \\
\hline Partner's Behavior: Generous (versus Coercive) & 1581.21 & 1 & 1581.21 & $53.29 * * *$ \\
\hline Computerized Partner X Generous Behavior & 13.15 & 1 & 13.15 & 0.44 \\
\hline Residual & 3264.02 & 110 & 29.67 & \\
\hline Adjusted R-Square & 0.31 & & & \\
\hline$* p<.05 ; * * p<.01 ; * * * p<.001$ (two-tailed). & & & & \\
\hline
\end{tabular}


Table 2. ANCOVA model Predicting Participant Rating of Shared Interests

\begin{tabular}{|l|l|l|l|l|}
\hline \multicolumn{1}{|c|}{ Source } & \multicolumn{1}{|c|}{ SS } & \multicolumn{1}{|c|}{ df } & \multicolumn{1}{|c|}{ MS } & \multicolumn{1}{|c|}{ F } \\
\hline Model & 303.94 & 3 & 101.31 & $155.81^{* * *}$ \\
\hline Type of Partner: Computer (versus Human) & 2.59 & 1 & 2.59 & $3.98^{*}$ \\
\hline Partner's Behavior: Generous (versus Selfish) & 302.21 & 1 & 302.21 & $464.77 * * *$ \\
\hline Computerized Partner X Generous Behavior & .05 & 1 & .05 & 0.08 \\
\hline Residual & 71.53 & 110 & .65 & \\
\hline Adjusted R-Square & .80 & & & \\
\hline$*$ * $p<.05 ; * * p<.01 ; * * * p<.001$ (two-tailed). & & & & \\
\hline
\end{tabular}


Table 3. ANCOVA model Predicting Participant Rating of Commitment

\begin{tabular}{|l|l|l|l|l|}
\hline \multicolumn{1}{|c|}{ Source } & \multicolumn{1}{|c|}{ SS } & \multicolumn{1}{c|}{ df } & \multicolumn{1}{c|}{ MS } & \multicolumn{1}{c|}{ F } \\
\hline Model & 156.20 & 3 & 52.07 & $61.47 * * *$ \\
\hline Type of Partner: Computer (versus Human) & 3.73 & 1 & 3.73 & $4.41^{*}$ \\
\hline Partner's Behavior: Generous (versus Selfish) & 153.61 & 1 & 153.61 & $181.34^{* * *}$ \\
\hline Computerized Partner X Generous Behavior & .02 & 1 & .02 & .03 \\
\hline Residual & 93.17 & 110 & .85 & \\
\hline Adjusted R-Square & .62 & & & \\
\hline$*$ p $<.05 ; * * p<.01 ; * * * p<.001$ (two-tailed). & & & & \\
\hline
\end{tabular}


Acknowledgements

This research was funded by the National Science Foundation under Grant No. SES-1264188. 


\section{HIGHLIGHTS}

- An experiment where participants worked with a partner they thought was a computer or human-being

- Participants were equally as generous to the computer and human partner

- These participants also reported higher levels of commitment with computers than humans

- Participants also thought their computer was more likely to have their best interests in mind than humans

- The results show how telling end-users that their partners are computers could improve how they perceive these interactions 